\title{
On a numerical quasiconformal mapping method for the medium parameters identification using applied quasipotential tomography
}

\author{
Bomba A., Boichura M. \\ Rivne State Humanitarian University \\ 12 Stepan Bandera str., 33000, Rivne, Ukraine
}

(Received 25 June 2017)

\begin{abstract}
The problem of parameters identification of the bursts of the medium conductivity coefficient according to the tomography of the applied quasipotentials is considered. The method of image reconstruction is suggested, according to which the problem of analysis is reduced to the application of numerical methods of quasiconformal mappings, and the problem of synthesis is reduced to the solving the problem of parametric identification. The results of numerical experiments are presented and their analysis is carried out.
\end{abstract}

Keywords: applied quasipotential tomography, quasiconformal mappings, identification, nonlinear problems.

2000 MSC: 30C30; 30C62

UDC: 519.6

\section{Introduction}

At present, there is a large number of image reconstruction methods meant for many types of subjects of inquiry (in industry, geophysics, medicine, etc.) and for obtaining various kinds of information about them. The main criteria that characterize this or that approach include complexity, harmfulness, resolution, and price. One of the most simple, safe, cheap, and flexible visualization methods is the Electrical Impedance Tomography. The idea of this method is to determine the distribution of the internal conductivity of the subject being investigated according to the measurements data on its boundary. This, in particular, makes it possible to conduct noninvasive studies.

A considerable number of works both foreign and domestic scientists are dedicated to the issue of the development of effective methods for the reconstruction of images in solving the electrical impedance tomography tasks (see, for example [1-13]). In most cases, various assumptions about the distribution of the conductivity coefficient (CC) in an interior of the investigated area (homogeneity, piecewise homogeneity with the infinite or the given finite number of values, heterogeneity, piecewise heterogeneity, anisotropy, the possibility of separating variables, etc.) are advanced. In particular, in [3] the medium is considered to be a piecewise-homogeneous one; the identification is carried out using a specially developed procedure for "averaging" the intermediate iterative approximations of the CC based on the data for all injections. In [1], it is proposed to reconstruct an image using the ideas of linearization (functions of potentials and costs) and regularization. However, here the CC distribution function depends on the coordinates of the domain of complex quasipotential, which, in its turn, narrows the applicability of the approach only to cases of specific physical processes. Other restrictions are available in [2], where it is assumed that the required coefficient allows for the separation of variables.

The purpose of this paper is to extend the suggested in [14] method of applied quasipotentials over the case of identification of the CC in the presence of smooth local bursts in homogeneous materials (in the form of "traditional caps"). In this case, in particular, we set the task to estimate the relationship between the number of injections and the quality of the reconstruction. 


\section{The problem statement}

Consider quasiideal processes of motion of particles (in particular, electric charges) in a simply connected curvilinear domain (plate, tomographic section, etc.) $G_{z}$ (Fig. 1a, where the internal curves are lines of the corresponding dynamic grid), bounded with a smooth closed curve $\partial G_{z}=\{(x, y): x=$ $\tilde{x}(\tau), y=\tilde{y}(\tau), 0 \leqslant \tau \leqslant 2 \pi, \tilde{x}(0)=\tilde{x}(2 \pi)=\tilde{x}_{0}, \tilde{y}(0)=\tilde{y}(2 \pi)=\tilde{y}_{0}$, where $\tilde{x}(\tau), \tilde{y}(\tau)$ are certain continuously differentiated functions, $O\left(\tilde{x}_{0}, \tilde{y}_{0}\right)$ is the given reference point.

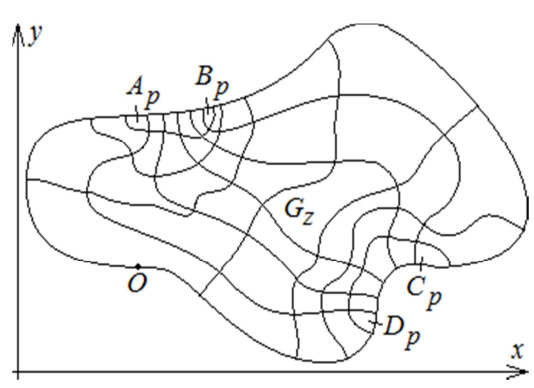

$a$

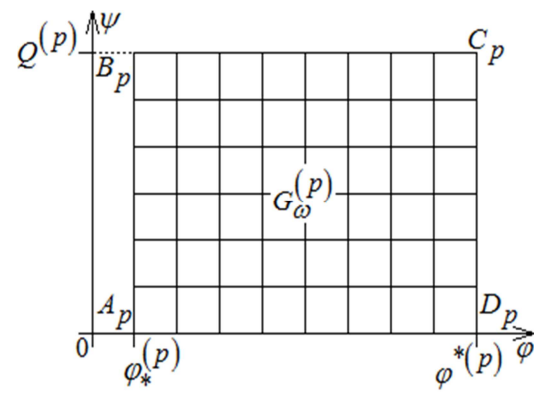

$b$

Fig. 1. Tomographic cross section $G_{z}(a)$, corresponding area of the complex quasipotential $G_{\omega}^{(p)}(b)$.

We assume that the motion of the electric charges in the area $G_{z}$ is realised according to a linear law such as Ohm's law, Darcy's law, etc. $\boldsymbol{j}^{(p)}=\sigma \operatorname{grad} \varphi^{(p)}$ under the conditions $\operatorname{div} \boldsymbol{j}^{(p)}=0$ (continuity), $\left.\varphi\right|_{A_{p} B_{p}}=\varphi_{*}^{(p)},\left.\varphi\right|_{C_{p} D_{p}}=\varphi^{*(p)}\left(\varphi^{*(p)}-\varphi_{*}^{(p)}>0, \varphi_{*}^{(p)}, \varphi^{*(p)}\right.$ are the applied quasipotentials $)$ and $\left.\frac{d \varphi^{(p)}}{d n}\right|_{B_{p} C_{p}}=\left.\frac{d \varphi^{(p)}}{d n}\right|_{A_{p} D_{p}}=0$ (nonpermittivity), $\left.\varphi^{(p)}(M)\right|_{B_{p} C_{p}}=\bar{\varphi}^{(p)}(M),\left.\varphi^{(p)}(M)\right|_{A_{p} D_{p}}=\underline{\varphi}^{(p)}(M)$, $\sigma \partial \varphi^{(p)}(M) /\left.\partial n\right|_{A_{p} B_{p}}=\psi_{*}^{(p)}(M), \sigma \partial \varphi^{(p)}(M) /\left.\partial n\right|_{C_{p} D_{p}}=\psi^{*(p)}(M)$ (additional conditions) [4], where $M$ is a running point of the corresponding curve; $\boldsymbol{j}^{(p)}=\left(j_{x}^{(p)}(x, y), j_{y}^{(p)}(x, y)\right.$ ) is the density (local velocity) of current; $\varphi^{(p)}=\varphi^{(p)}(x, y)$ is the quasipotential of the field such as $A_{p} B_{p}$ and $C_{p} D_{p}$ are the equipotential lines; $B_{p} C_{p}$ and $A_{p} D_{p}$ are the impenetrable boundary stream lines; $A_{p}, B_{p}, C_{p}, D_{p}$ are the denoted points on $\partial G_{z} ; p=1,2, \ldots$ is a parameter-number of injections (see, e.g. [1,3,17]); $\boldsymbol{n}$ is an external normal to the corresponding section of the boundary of this area [14]; $\psi^{(p)}=\psi^{(p)}(x, y)$ is the function of stream (so we have, so called, a model of shunt $[1,3,7,17]$ ). The functions $\bar{\varphi}^{(p)}(M)=$ $\bar{\varphi}^{(p)}(\tau, \ldots)\left(\tau_{C}^{(p)} \leqslant \tau \leqslant \tau_{B}^{(p)}\right), \underline{\varphi}^{(p)}(M)=\underline{\varphi}^{(p)}(\tau, \ldots)\left(\tau_{A}^{(p)} \leqslant \tau \leqslant \tau_{D}^{(p)}\right), \psi_{*}^{(p)}(M)=\psi_{*}^{(p)}(\tau, \ldots)\left(\tau_{B}^{(p)} \leqslant\right.$ $\left.\tau \leqslant \tau_{A}^{(p)}\right), \psi^{*(p)}(M)=\psi^{*(p)}(\tau, \ldots)\left(\tau_{D}^{(p)} \leqslant \tau \leqslant \tau_{C}^{(p)}\right)$, as in [3], can be constructed by interpolating experimentally obtained values $\bar{\varphi}_{\bar{i}^{(p)}}^{(p)}, \underline{\varphi}_{\underline{\underline{i}}^{(p)}}^{(p)}, \psi_{*_{j_{*}^{*}}^{(p)}}^{(p)}, \psi_{j^{*(p)}}^{*(p)}$ for some $\tau=\bar{\tau}_{\bar{i}^{(p)}}^{(p)}, \tau={\underline{\tau_{\underline{i}}}}^{(p)}, \tau=\tau_{* j_{*}^{(p)}}^{(p)}$, $\tau=\tau_{j^{*(p)}}^{*(p)}$ in the intervals $B_{p} C_{p}, A_{p} D_{p}, A_{p} B_{p}, C_{p} D_{p}$, correspondingly $\left(\varphi_{*}^{(p)} \leqslant \underline{\varphi}_{\underline{\underline{i}}(p)}^{(p)} \leqslant \varphi^{*(p)}, \varphi_{*}^{(p)} \leqslant\right.$ $\bar{\varphi}_{\bar{i}^{(p)}}^{(p)} \leqslant \varphi^{*(p)}, \psi_{* j_{*}^{(p)}}^{(p)}>0, \psi_{j^{*(p)}}^{*(p)}>0,0 \leqslant \bar{i}^{(p)} \leqslant \bar{m}^{*(p)}+1,0 \leqslant \underline{i}^{(p)} \leqslant \underline{m}_{*}^{(p)}+1,0 \leqslant j_{*}^{(p)} \leqslant n_{*}^{(p)}+1$, $\left.0 \leqslant j^{*(p)} \leqslant n^{*(p)}+1\right)$. The CC being searched for in the form of a sum of its main part and local bursts, namely:

$$
\sigma\left(x, y, \chi, \alpha_{1}, \ldots, \alpha_{s}, \varepsilon_{1}, \ldots, \varepsilon_{s}, x_{1}, \ldots, x_{s}, y_{1}, \ldots, y_{s}\right)=\chi+\sum_{k=1}^{s} \frac{\alpha_{k}}{1+\left(\left(x-x_{k}\right)^{2}+\left(y-y_{k}\right)^{2}\right) / \varepsilon_{k}},
$$

where $\chi, \alpha_{k}, \varepsilon_{k}, x_{k}, y_{k}(k=1, \ldots, s)$ are parameters to be found in the course of solving the problem.

The injections of current (with the corresponding physical measurements) through a tomographic cross section will be modeled (in the first place) by sets of the values $\left\{\tau_{A}^{(p)}, \tau_{B}^{(p)}, \tau_{C}^{(p)}, \tau_{D}^{(p)}\right\}$, according to which, $A_{p}=\left(\tilde{x}\left(\tau_{A}^{(p)}\right), \tilde{y}\left(\tau_{A}^{(p)}\right)\right), B_{p}=\left(\tilde{x}\left(\tau_{B}^{(p)}\right), \tilde{y}\left(\tau_{B}^{(p)}\right)\right), C_{p}=\left(\tilde{x}\left(\tau_{C}^{(p)}\right), \tilde{y}\left(\tau_{C}^{(p)}\right)\right), D_{p}=\left(\tilde{x}\left(\tau_{D}^{(p)}\right), \tilde{y}\left(\tau_{D}^{(p)}\right)\right)$ (the corresponding boundary of the area $G_{z}$ with the given 4 denoted points we indicate by $\left.\partial G_{z}^{(p)}\right)$.

The task consists in calculating electrodynamic grids, velocity fields, and in reconstructing the CC image. 


\section{Problems of analysis and synthesis}

Introducing the stream function $\psi^{(p)}(x, y)$, which are complex conjugate to the functions $\varphi^{(p)}(x, y)$ $(p=\overline{1, \tilde{p}})$, by analogy with [1-3,14-16], we arrive at a series of more general boundary-value problems on quasiconformal mappings $\omega=\omega^{(p)}(z)=\varphi^{(p)}(x, y)+i \psi^{(p)}(x, y)$ of physical areas $G_{z}^{(p)}$ (Fig. 1a) to the corresponding areas of the complex quasipotential $G_{\omega}^{(p)}$ (Fig. 1b):

$$
\begin{gathered}
\sigma \frac{\partial \varphi^{(p)}}{\partial x}=\frac{\partial \psi^{(p)}}{\partial y}, \quad \sigma \frac{\partial \varphi^{(p)}}{\partial y}=-\frac{\partial \psi(p)}{\partial x} \\
\left.\varphi^{(p)}\right|_{A_{p} B_{p}}=\varphi_{*}^{(p)},\left.\quad \varphi^{(p)}\right|_{C_{p} D_{p}}=\varphi^{*(p)},\left.\quad \psi^{(p)}\right|_{A_{p} D_{p}}=0,\left.\quad \psi^{(p)}\right|_{B_{p} C_{p}}=Q^{(p)} ; \\
\int_{M N} \sigma \frac{\partial \varphi^{(p)}}{\partial n} d l=Q^{(p)}, \quad M \in B_{p} C_{p}, \quad N \in A_{p} D_{p} ; \\
\left.\varphi^{(p)}(M)\right|_{B_{p} C_{p}}=\bar{\varphi}^{(p)}(M),\left.\quad \varphi^{(p)}(M)\right|_{A_{p} D_{p}}=\underline{\varphi}^{(p)}(M), \\
\left.\psi^{(p)}(M)\right|_{A_{p} B_{p}}=\Psi_{*}^{(p)}(M),\left.\quad \psi^{(p)}(M)\right|_{C_{p} D_{p}}=\Psi^{*(p)}(M),
\end{gathered}
$$

where $G_{\omega}^{(p)}=\left\{(\varphi, \psi): \varphi_{*}^{(p)} \leqslant \varphi \leqslant \varphi^{(p) *}, 0 \leqslant \psi \leqslant Q^{(p)}\right\} ; Q^{(p)}$ are flows of the vector field (current) through the contact surface $\left(A_{p} B_{p}\right.$ and $\left.C_{p} D_{p}\right), \Psi_{*}^{(p)}(M)=\int_{A_{p} M} \psi_{*}^{(p)}(M) d l, \Psi^{*(p)}(M)=$ $\int_{D_{p} M} \psi^{*(p)}(M) d l ; d l$ is the element of an arc of the corresponding curve.

The work [14] substantiates the expediency of the transition from the direct problems (on quasiconformal mappings $G_{z}$ to $\left.G_{\omega}\right)$ to the inverse ones. The inverse nonlinear boundary value problems on quasiconformal mappings $z=z^{(p)}(\omega)=x^{(p)}(\varphi, \psi)+i y^{(p)}(\varphi, \psi)$ of the areas $G_{\omega}^{(p)}$ to $G_{z}^{(p)}$ with the unknowns $\varphi^{(p)}(x, y)$ and $\psi^{(p)}(x, y)$ by analogy with $[1-3,14,15]$, we reduce to the solving the system of elliptic equations:

$$
\left\{\begin{array}{l}
\frac{\partial}{\partial \varphi}\left(\frac{1}{\sigma} \frac{\partial x^{(p)}}{\partial \varphi}\right)+\frac{\partial}{\partial \psi}\left(\sigma \frac{\partial x^{(p)}}{\partial \psi}\right)=0 \\
\frac{\partial}{\partial \varphi}\left(\frac{1}{\sigma} \frac{\partial y^{(p)}}{\partial \varphi}\right)+\frac{\partial}{\partial \psi}\left(\sigma \frac{\partial y^{(p)}}{\partial \psi}\right)=0, \quad \forall\left(x^{(p)}, y^{(p)}\right) \in G_{z}^{(p)}
\end{array}\right.
$$

under the conditions:

$$
\begin{gathered}
x^{(p)}\left(\varphi_{*}^{(p)}, \psi\right)=\tilde{x}\left(\tau_{*}^{(p)}(\psi)\right), \quad y^{(p)}\left(\varphi_{*}^{(p)}, \psi\right)=\tilde{y}\left(\tau_{*}^{(p)}(\psi)\right), \\
x^{(p)}\left(\varphi, Q^{(p)}\right)=\tilde{x}\left(\bar{\tau}^{(p)}(\varphi)\right), \quad y^{(p)}\left(\varphi, Q^{(p)}\right)=\tilde{y}\left(\bar{\tau}^{(p)}(\varphi)\right), \\
x^{(p)}\left(\varphi^{*(p)}, \psi\right)=\tilde{x}\left(\tau^{*(p)}(\psi)\right), \quad y^{(p)}\left(\varphi^{*(p)}, \psi\right)=\tilde{y}\left(\tau^{*(p)}(\psi)\right), \\
\left.x^{(p)}(\varphi, 0)=\tilde{x} \underline{\tau}^{(p)}(\varphi)\right), \quad y^{(p)}(\varphi, 0)=\tilde{y}\left(\underline{\tau}^{(p)}(\varphi)\right) ; \\
\left\{\begin{array}{cc}
\tilde{x}^{\prime}(\tau) \frac{\partial y^{(p)}\left(\varphi_{*}^{(p)}, \psi_{*}^{(p)}(\tau, \ldots)\right)}{\partial \varphi}-\tilde{y}^{\prime}(\tau) \frac{\partial x^{(p)}\left(\varphi_{*}^{(p)}, \psi_{*}^{(p)}(\tau, \ldots)\right)}{\partial \varphi}=0, & \tau \in\left[\tau_{B}^{(p)} ; \tau_{A}^{(p)}\right], \\
\tilde{x}^{\prime}(\tau) \frac{\partial y^{(p)}\left(\varphi^{*(p)}, \psi^{*(p)}(\tau, \ldots)\right)}{\partial \varphi}-\tilde{y}^{\prime}(\tau) \frac{\partial x^{(p)}\left(\varphi^{*(p)}, \psi^{*(p)}(\tau, \ldots)\right)}{\partial \varphi}=0, & \tau \in\left[\tau_{D}^{(p)} ; \tau_{C}^{(p)}\right], \\
\tilde{x}^{\prime}(\tau) \frac{\partial y^{(p)}\left(\underline{\varphi}^{(p)}(\tau, \ldots), 0\right)}{\partial \psi}-\tilde{y}^{\prime}(\tau) \frac{\partial x^{(p)}\left(\underline{\varphi}^{(p)}(\tau, \ldots), 0\right)}{\partial \psi}=0, & \tau \in\left[\tau_{A}^{(p)} ; \tau_{D}^{(p)}\right], \\
\tilde{x}^{\prime}(\tau) \frac{\partial y^{(p)}\left(\bar{\varphi}^{(p)}(\tau, \ldots), Q^{(p)}\right)}{\partial \psi}-\tilde{y}^{\prime}(\tau) \frac{\partial x^{(p)}\left(\bar{\varphi}^{(p)}(\tau, \ldots), Q^{(p)}\right)}{\partial \psi}=0, & \tau \in\left[\tau_{C}^{(p)} ; \tau_{B}^{(p)}\right],
\end{array}\right.
\end{gathered}
$$


where $\tau=\tau_{*}^{(p)}(\psi), \tau=\bar{\tau}^{(p)}(\varphi), \tau=\tau^{*(p)}(\psi), \tau=\underline{\tau}^{(p)}(\varphi)\left(\varphi_{*}^{(p)} \leqslant \varphi \leqslant \varphi^{*(p)}, 0 \leqslant \psi \leqslant Q^{(p)}\right)$ are functions constructed by interpolating their experimentally obtained values $\tau_{* j_{*}}^{(p)}, \bar{\tau}_{\bar{i}}^{(p)}, \tau_{j^{*}}^{*(p)}, \underline{\tau}_{\underline{i}}^{(p)}$ at some arguments $\Psi_{* j_{*}}^{(p)}, \bar{\varphi}_{\bar{i}}^{(p)}, \Psi_{j^{*}}^{*(p)}, \underline{\varphi}_{i}^{(p)}$ in the intervals $A_{p} B_{p}, B_{p} C_{p}, C_{p} D_{p}$ and $A_{p} D_{p}$, correspondingly.

Let us reconstruct the $\mathrm{CC}$, provided that the functional is minimized

$$
\begin{aligned}
& \Phi\left(x^{(1)}, \ldots, x^{(\tilde{p})}, y^{(1)}, \ldots, y^{(\tilde{p})}, \chi, \alpha_{1}, \ldots, \alpha_{s}, \varepsilon_{1}, \ldots, \varepsilon_{s}, x_{1}, \ldots, x_{s}, y_{1}, \ldots, y_{s}\right) \\
& \quad=\sum_{p=1}^{\tilde{p}}\left(\sigma\left(x^{(p)}, y^{(p)}, \chi, \alpha_{1}, \ldots, \alpha_{s}, \varepsilon_{1}, \ldots, \varepsilon_{s}, x_{1}, \ldots, x_{s}, y_{1}, \ldots, y_{s}\right)-f\left(x^{(p)}, y^{(p)}\right)\right)^{2} \rightarrow \min ,
\end{aligned}
$$

where $f\left(x^{(p)}, y^{(p)}\right)=\left(\left(\frac{\partial x^{(p)}}{\partial \varphi}\right)^{2}+\left(\frac{\partial y^{(p)}}{\partial \varphi}\right)^{2}\right)^{1 / 2}\left(\left(\frac{\partial x^{(p)}}{\partial \psi}\right)^{2}+\left(\frac{\partial y^{(p)}}{\partial \psi}\right)^{2}\right)^{-1 / 2}$

\section{The difference analogue of the problem}

The difference analogue of the problem (5)-(8) in the uniform grid domains $G_{\omega}^{\gamma(p)}$ is obtained analogously to $[1-3,14,20]$.

Using the "cross" scheme, the difference analogues of the elliptic equations (5) in the grid domains $G_{\omega}^{\gamma(p)}$ can be represented as follows

$$
\left\{\begin{aligned}
x_{i, j}^{(p)}= & \frac{1}{2\left(1+\gamma^{(p) 2} \sigma_{i, j}^{\gamma(p) 2}\right)}\left(x_{i+1, j}^{(p)}+x_{i-1, j}^{(p)}+\gamma^{(p) 2} \sigma_{i, j}^{\gamma(p) 2}\left(x_{i, j-1}^{(p)}+x_{i, j+1}^{(p)}\right)\right. \\
& +\frac{\gamma^{(p) 2} \sigma_{i, j}^{\gamma(p)}}{4}\left(\frac{\partial \sigma_{i, j}^{\gamma(p)}}{\partial x}\left(x_{i, j+1}^{(p)}-x_{i, j-1}^{(p)}\right)+\frac{\partial \sigma_{i, j}^{\gamma(p)}}{\partial y}\left(y_{i, j+1}^{(p)}-y_{i, j-1}^{(p)}\right)\right)\left(x_{i, j+1}^{(p)}-x_{i, j-1}^{(p)}\right) \\
& \left.-\frac{1}{4 \sigma_{i, j}^{\gamma(p)}}\left(\frac{\partial \sigma_{i, j}^{\gamma(p)}}{\partial x}\left(x_{i+1, j}^{(p)}-x_{i-1, j}^{(p)}\right)+\frac{\partial \sigma_{i, j}^{\gamma(p)}}{\partial y}\left(y_{i+1, j}^{(p)}-y_{i-1, j}^{(p)}\right)\right)\left(x_{i+1, j}^{(p)}-x_{i-1, j}^{(p)}\right)\right) \\
y_{i, j}^{(p)}= & \frac{1}{2\left(1+\gamma^{(p) 2} \sigma_{i, j}^{\gamma(p) 2}\right)}\left(y_{i+1, j}^{(p)}+y_{i-1, j}^{(p)}+\gamma^{(p) 2} \sigma_{i, j}^{\gamma(p) 2}\left(y_{i, j-1}^{(p)}+y_{i, j+1}^{(p)}\right)\right. \\
& +\frac{\gamma^{(p) 2} \sigma_{i, j}^{\gamma(p)}}{4}\left(\frac{\partial \sigma_{i, j}^{\gamma(p)}}{\partial x}\left(x_{i, j+1}^{(p)}-x_{i, j-1}^{(p)}\right)+\frac{\partial \sigma_{i, j}^{\gamma(p)}}{\partial y}\left(y_{i, j+1}^{(p)}-y_{i, j-1}^{(p)}\right)\right)\left(y_{i, j+1}^{(p)}-y_{i, j-1}^{(p)}\right) \\
& \left.-\frac{1}{4 \sigma_{i, j}^{\gamma(p)}}\left(\frac{\partial \sigma_{i, j}^{\gamma(p)}}{\partial x}\left(x_{i+1, j}^{(p)}-x_{i-1, j}^{(p)}\right)+\frac{\partial \sigma_{i, j}^{\gamma(p)}}{\partial y}\left(y_{i+1, j}^{(p)}-y_{i-1, j}^{(p)}\right)\right)\left(y_{i+1, j}^{(p)}-y_{i-1, j}^{(p)}\right)\right),
\end{aligned}\right.
$$

where $\gamma^{(p)}$ are the quasiconformal invariants [14] for the corresponding domains $G_{\omega}^{\gamma(p)}=$ $\left\{\left(\varphi_{i}^{(p)}, \psi_{j}^{(p)}\right): \varphi_{i}^{(p)}=\varphi_{*}^{(p)}+i \Delta \varphi^{(p)}, i=\overline{0, m^{(p)}+1} ; \psi_{j}^{(p)}=j \Delta \psi^{(p)}, j=\overline{0, n^{(p)}+1} ; \Delta \varphi^{(p)}=\frac{\varphi^{(p) *}-\varphi_{*}^{(p)}}{m^{(p)}+1}\right.$, $\left.\Delta \psi^{(p)}=\frac{Q^{(p)}}{n^{(p)}+1}, \gamma^{(p)}=\frac{\Delta \varphi^{(p)}}{\Delta \psi^{(p)}}, m^{(p)}, n^{(p)} \in N\right\} ; x_{i, j}^{(p)}=x^{(p)}\left(\varphi_{i}^{(p)}, \psi_{j}^{(p)}\right), y_{i, j}^{(p)}=y^{(p)}\left(\varphi_{i}^{(p)}, \psi_{j}^{(p)}\right)$, $\sigma_{i, j \pm 1 / 2}^{\gamma(p)}=\sigma\left(\left(x_{i, j \pm 1}^{(p)}+x_{i, j}^{(p)}\right) / 2,\left(y_{i, j \pm 1}^{(p)}+y_{i, j}^{(p)}\right) / 2\right), \sigma_{i \pm 1 / 2, j}^{\gamma(p)}=\sigma\left(\left(x_{i \pm 1, j}^{(p)}+x_{i, j}^{(p)}\right) / 2,\left(y_{i \pm 1, j}^{(p)}+y_{i, j}^{(p)}\right) / 2\right)$, $\left(x_{i, j}^{(p)}, y_{i, j}^{(p)}\right) \in G_{z}^{(p)}$.

The conditions (3) are approximated by such point relations

$$
\begin{gathered}
x_{0, j}^{(p)}=\tilde{x}\left(\tau_{*}^{(p)}\left(\psi_{j}\right)\right), \quad y_{0, j}^{(p)}=\tilde{y}\left(\tau_{*}^{(p)}\left(\psi_{j}\right)\right), \quad x_{i, n^{(p)}+1}^{(p)}=\tilde{x}\left(\bar{\tau}^{(p)}\left(\varphi_{i}\right)\right), \\
y_{i, n^{(p)}+1}^{(p)}=\tilde{y}\left(\bar{\tau}^{(p)}\left(\varphi_{i}\right)\right), \quad x_{m^{(p)}+1, j}^{(p)}=\tilde{x}\left(\tau^{*(p)}\left(\psi_{j}\right)\right), \quad y_{m^{(p)}+1, j}^{(p)}=\tilde{y}\left(\tau^{*(p)}\left(\psi_{j}\right)\right),
\end{gathered}
$$

Mathematical Modeling and Computing, Vol.4, No. 1, pp. 10-20 (2017) 


$$
x_{i, 0}^{(p)}=\tilde{x}\left(\underline{\tau}^{(p)}\left(\varphi_{i}\right)\right), \quad y_{i, 0}^{(p)}=\tilde{y}\left(\underline{\tau}^{(p)}\left(\varphi_{i}\right)\right) \quad\left(0 \leqslant i \leqslant m^{(p)}+1, \quad 0 \leqslant j \leqslant n^{(p)}+1\right) .
$$

The conditions of orthogonality (7) on the boundary of the area are approximated by the following point and difference equations

$$
\left\{\begin{array}{l}
\tilde{x}^{\prime}(\tau)\left(y_{1, j}^{(p)}-y_{0, j}^{(p)}\right)-\tilde{y}^{\prime}(\tau)\left(x_{1, j}^{(p)}-x_{0, j}^{(p)}\right)=0, \quad \tau \in\left[\tau_{B}^{(p)} ; \tau_{A}^{(p)}\right] \\
\tilde{x}^{\prime}(\tau)\left(y_{m, j}^{(p)}-x_{m+1, j}^{(p)}\right)-\tilde{y}^{\prime}(\tau)\left(x_{m, j}^{(p)}-x_{m+1, j}^{(p)}\right)=0, \quad \tau \in\left[\tau_{D}^{(p)} ; \tau_{C}^{(p)}\right] \\
\tilde{x}^{\prime}(\tau)\left(y_{i, n}^{(p)}-x_{i, n+1}^{(p)}\right)-\tilde{y}^{\prime}(\tau)\left(x_{i, n}^{(p)}-x_{i, n+1}^{(p)}\right)=0, \quad \tau \in\left[\tau_{C}^{(p)} ; \tau_{B}^{(p)}\right] \\
\tilde{x}^{\prime}(\tau)\left(y_{i, 1}^{(p)}-y_{i, 0}^{(p)}\right)-\tilde{y}^{\prime}(\tau)\left(x_{i, 1}^{(p)}-x_{i, 0}^{(p)}\right)=0, \quad \tau \in\left[\tau_{A}^{(p)} ; \tau_{D}^{(p)}\right] \\
\left(1 \leqslant i \leqslant m^{(p)}, \quad 1 \leqslant j \leqslant n^{(p)}\right) .
\end{array}\right.
$$

The functional (8) we rewrite for all internal nodal points $\left(x_{i, j}^{(p)}, y_{i, j}^{(p)}\right)\left(p=\overline{1, \tilde{p}}, i=\overline{1, m^{(p)}}, j=\right.$ $\left.\overline{1, n^{(p)}}\right)$ as follows

$$
\begin{array}{r}
\Phi\left(x^{(1)}, \ldots, x^{(\tilde{p})}, y^{(1)}, \ldots, y^{(\tilde{p})}, \chi, \alpha_{1}, \ldots, \alpha_{s}, \varepsilon_{1}, \ldots, \varepsilon_{s}, x_{1}, \ldots, x_{s}, y_{1}, \ldots, y_{s}\right) \\
=\sum_{p=1}^{\tilde{p}} \sum_{i=1}^{m^{(p)}} \sum_{j=1}^{n^{(p)}}\left(\sigma\left(x_{i, j}^{(p)}, y_{i, j}^{(p)}, \chi, \alpha_{1}, \ldots, \alpha_{s}, \varepsilon_{1}, \ldots, \varepsilon_{s}, x_{1}, \ldots, x_{s}, y_{1}, \ldots, y_{s}\right)\right. \\
\left.-f^{\gamma}\left(x_{i-1, j-1}^{(p)}, y_{i-1, j+1}^{(p)}, x_{i+1, j-1}^{(p)}, y_{i+1, j+1}^{(p)}\right)\right)^{2} \rightarrow \min
\end{array}
$$

where

$$
\begin{aligned}
f^{\gamma}\left(x_{i-1, j-1}^{(p)}, y_{i-1, j+1}^{(p)},\right. & \left.x_{i+1, j-1}^{(p)}, y_{i+1, j+1}^{(p)}\right)=\left(\left(\left(x_{i+1, j-1}^{(p)}-x_{i-1, j-1}^{(p)}\right)^{2}+\left(y_{i+1, j-1}^{(p)}-y_{i-1, j-1}^{(p)}\right)^{2}\right)^{0.5}\right. \\
+ & \left.\left(\left(x_{i+1, j+1}^{(p)}-x_{i-1, j+1}^{(p)}\right)^{2}+\left(y_{i+1, j+1}^{(p)}-y_{i-1, j+1}^{(p)}\right)^{2}\right)^{0.5}\right) \\
& \times\left(\left(\left(x_{i-1, j+1}^{(p)}-x_{i-1, j-1}^{(p)}\right)^{2}+\left(y_{i-1, j+1}^{(p)}-y_{i-1, j-1}^{(p)}\right)^{2}\right)^{0.5}\right. \\
& \left.+\left(\left(x_{i+1, j+1}^{(p)}-x_{i+1, j-1}^{(p)}\right)^{2}+\left(y_{i+1, j+1}^{(p)}-y_{i+1, j-1}^{(p)}\right)^{2}\right)^{0.5}\right)^{-1} \frac{1}{\gamma^{(p)}} .
\end{aligned}
$$

\section{Solving algorithm}

The algorithm for solving the initial problem consists in the alternate parameterization of the internal nodes of the grid domains $G_{z}^{\gamma(p)}$, the CC, and using the ideas of the method of block iteration [18]. Namely: we set the number of injections $\tilde{p}$, the boundary of the domains $G_{z}^{(p)}$ (by the functions $x=\tilde{x}(\tau), y=\tilde{y}(\tau))$, the parameters $\tau_{A}^{(p)}, \tau_{B}^{(p)}, \tau_{C}^{(p)}, \tau_{D}^{(p)}, q(q>1$ is responsible for the number of iterations of the refinement on internal nodes for a specific CC), the quasipotentials $\varphi_{*}^{(p)}, \varphi^{*(p)}$, and losses $Q^{(p)}, m^{(p)}, n^{(p)}$ are the parameters of the partition of the domains $G_{\omega}^{\gamma(p)}$ (preferably so that $\left.\frac{Q^{(p)}}{\varphi^{*(p)}-\varphi_{*}^{(p)}} \frac{n^{(p)}+1}{m^{(p)}+1} \approx 1\right)$. With this, we calculate the coordinates of the angular points $A_{p}=$ $\left(\tilde{x}\left(\tau_{A}^{(p)}\right), \tilde{y}\left(\tau_{A}^{(p)}\right)\right), B_{p}=\left(\tilde{x}\left(\tau_{B}^{(p)}\right), \tilde{y}\left(\tau_{B}^{(p)}\right)\right), C_{p}=\left(\tilde{x}\left(\tau_{C}^{(p)}\right), \tilde{y}\left(\tau_{C}^{(p)}\right)\right), D_{p}=\left(\tilde{x}\left(\tau_{D}^{(p)}\right), \tilde{y}\left(\tau_{D}^{(p)}\right)\right)$ on $\partial G_{z}^{(p)}$, $\Delta \varphi^{(p)}=\frac{\varphi^{*(p)}-\varphi_{*}^{(p)}}{m^{(p)}+1}, \Delta \psi^{(p)}=\frac{Q^{(p)}}{n^{(p)}+1}$ and the values of quasiconformal invariants $\gamma^{(p)}=\Delta \varphi^{(p)} / \Delta \psi^{(p)}$. 
Setting the values of the stream functions $\psi_{* j}^{(p)}, \psi_{j}^{*(p)}$ and the potentials $\bar{\varphi}_{i}^{(p)}, \underline{\varphi}_{i}^{(p)}$ for some arguments $\tau_{* j}^{(p)}, \tau_{j}^{*(p)}, \bar{\tau}_{i}^{(p)}, \underline{\tau}_{i}^{(p)}$ (results of physical measurements), respectively, by means of interpolation we calculate (10), after that we find the coordinates of the nodes $x_{0, j}^{(p)}, y_{0, j}^{(p)}, x_{i, n^{(p)}+1}^{(p)}, y_{i, n^{(p)}+1}^{(p)}, x_{m^{(p)}+1, j}^{(p)}$, $y_{m(p)+1, j}^{(p)}, x_{i, 0}^{(p)}, y_{i, 0}^{(p)}\left(0 \leqslant i \leqslant m^{(p)}+1,0 \leqslant j \leqslant n^{(p)}+1, p=\overline{1, \tilde{p}}\right)$ on $\partial G_{z}^{(p)}$. The initial approximations $x_{i, j}^{(p, 0)}, y_{i, j}^{(p, 0)}$ and parameters $\chi^{(0)}, \alpha_{k}^{(0)}, \varepsilon_{k}^{(0)}, x_{k}^{(0)}, y_{k}^{(0)}(k=1, \ldots, s)$, which define the CC, we form, for example, as follows: $x_{i, j}^{(p, 0)}=\left(x_{i, 0}^{(p)}+x_{i, n^{(p)}}^{(p)}+x_{0, j}^{(p)}+x_{m^{(p)}, j}^{(p)}\right) / 4, y_{i, j}^{(p, 0)}=\left(y_{i, 0}^{(p)}+y_{i, n}^{(p)}+y_{0, j}^{(p)}+y_{m^{(p)}, j}^{(p)}\right) / 4$ $\left(1 \leqslant i \leqslant m^{(p)}, 1 \leqslant j \leqslant n^{(p)}, p=\overline{1, \tilde{p}}\right), \chi^{(0)}=1, \alpha_{k}^{(0)}=0, \varepsilon_{k}^{(0)}=1, x_{k}^{(0)}=0, y_{k}^{(0)}=0(k=1, \ldots, s)$.

The iterative process of reconstruction consists of the following steps: refine on the coordinates of the boundary nodes (using the difference analogues of the Cauchy-Riemann type) by solving the system of equations [3]

$$
\begin{gathered}
\left\{\begin{array}{c}
x_{1, j}^{(p, l+1)}=x_{0, j}+0.5 \gamma^{(p)} \sigma_{0, j}^{(l)}\left(y_{0, j+1}^{(p, l)}-y_{0, j-1}^{(p, l)}\right), \\
y_{1, j}^{(p, l+1)}=y_{0, j}-0.5 \gamma^{(p)} \sigma_{0, j}^{(l)}\left(x_{0, j+1}^{(p, l)}-x_{0, j-1}^{(p, l)}\right) ;
\end{array}\right. \\
\left\{\begin{array}{l}
x_{m, j}^{(p, l+1)}=x_{m+1, j}-0.5 \gamma^{(p)} \sigma_{m+1, j}^{(l)}\left(y_{m+1, j+1}^{(p, l)}-y_{m+1, j-1}^{(p, l)}\right), \\
y_{m, j}^{(p, l+1)}=y_{m+1, j}+0.5 \gamma^{(p)} \sigma_{m+1, j}^{(l)}\left(x_{m+1, j+1}^{(p, l)}-x_{m+1, j-1}^{(p, l)}\right) ;
\end{array}\right. \\
\left\{\begin{array}{l}
x_{i, 1}^{(p, l+1)}=x_{i, 0}-0.5 \gamma^{(p)} \sigma_{i, 0}^{(l)}\left(y_{i+1,0}^{(p, l)}-y_{i-1,0}^{(p, l)}\right), \\
y_{i, 1}^{(p, l+1)}=y_{i, 0}+0.5 \gamma^{(p)} \sigma_{i, 0}^{(l)}\left(x_{i+1,0}^{(p, l)}-x_{i-1,0}^{(p, l)} ;\right.
\end{array}\right. \\
\left\{\begin{array}{c}
x_{i, n}^{(p, l+1)}=x_{i, n+1}+0.5 \gamma^{(p)} \sigma_{i, n+1}^{(l)}\left(y_{i+1, n+1}^{(p, l)}-y_{i-1, n+1}^{(p, l)}\right), \\
y_{i, n}^{(p, l+1)}=y_{i, n+1}-0.5 \gamma^{(p)} \sigma_{i, n+1}^{(l)}\left(x_{i+1, n+1}^{(p, l)}-x_{i-1, n+1}^{(p, l)}\right)
\end{array}\right.
\end{gathered}
$$

with respect to $\left(x_{1, j}^{(p, l+1)}, y_{1, j}^{(p, l+1)}\right),\left(x_{m, j}^{(p, l+1)}, y_{m, j}^{(p, l+1)}\right),\left(x_{i, 1}^{(p, l+1)}, y_{i, 1}^{(p, l+1)}\right),\left(x_{i, n}^{(p, l+1)}, y_{i, n}^{(p, l+1)}\right)$, respectively $\left(1 \leqslant i \leqslant m^{(p)}, 1 \leqslant j \leqslant n^{(p)}, 1 \leqslant p \leqslant \tilde{p}\right)$; according to (10) rifine on the coordinates of the internal nodes for $i=\overline{2, m^{(p)}-1}, j=\overline{2, n^{(p)}-1}, p=\overline{1, \tilde{p}}[1,3,14]$; repeat this procedure $q$ times; using one of the methods of global optimization (see, for example, [19]) minimize the functional (12) for the required $\chi^{(l)}, \alpha_{k}^{(l)}, \varepsilon_{k}^{(l)}, x_{k}^{(l)}, y_{k}^{(l)}$ (here $l=0,1, \ldots$ is the number of the itaeration step, $k=1, \ldots, s$ ); check the conditions for the completion of the iterative process, among which there can be $[3,14]$ the stabilization of the boundary nodes, the CC, the parameter of the degree of quasiconformality, the values of losses, etc. $\left(1 \leqslant p \leqslant \tilde{p}, 1 \leqslant i \leqslant m^{(p)}, 1 \leqslant j \leqslant n^{(p)}\right)$. In the case when at least one of these conditions is not satisfied, the iteration process is restarted, otherwise we construct the corresponding reconstructed image and, if it is necessary, electrodynamic grids, the domains of complex quasipotential, or calculate the fields of current densities by the formula $\boldsymbol{j}^{(p)}=\sigma(x, y) \cdot \operatorname{grad} \varphi^{(p)}$ etc.

It should be noted that here, as in [3] (in contrast to $[1,2,14,15]$ ), there is no need to use the formulas to refine on the boundary nodes (their coordinates are a priori known), which, of course, accelerates the process of reconstruction of an image. In order to save computing time, it is also allowed using the formula (12) only for the selected points.

In order to increase the accuracy of quasiconformal mappings (first of all, in the vicinity of the angular boundary points), it is possible to introduce a specially developed procedure of "replacing" the smoothness at the points $A_{p}, B_{p}, C_{p}, D_{p}$ with the orthogonality of the corresponding stream lines and the equipotential lines.

We also note that instead of giving the coordinates of the boundary nodes by the formula (10) (by interpolating the results of physical measurements), one can immediately select them so that the local differences in the values of the stream function or the potential between them at the corresponding neighbouring points are to be constant within the injection limits. 


\section{Numerical analysis}

Let us present the results of numerical analysis with $\tilde{x}(\tau)=150 \cos \tau, \tilde{y}(\tau)=100 \sin \tau, m^{(p)}=100$; $\tau_{A}^{(p)}=\frac{\pi}{8}+(p-1) \frac{\pi}{2 \tilde{p}}+\pi, \tau_{B}^{(p)}=\tau_{A}^{(p)}-\frac{\pi}{4}, \tau_{C}^{(p)}=\tau_{A}^{(p)}-\pi, \tau_{D}^{(p)}=\tau_{C}^{(p)}-\frac{\pi}{4} ; \varphi_{*}^{(p)}=0, \varphi^{*(p)}=1, \chi^{(0)}=1$, $\alpha_{k}^{(0)}=0, \varepsilon_{k}^{(0)}=1, x_{k}^{(0)}=0, y_{k}^{(0)}=0,0.1 \leqslant \chi \leqslant 1.5,0 \leqslant \alpha_{k} \leqslant 2,10^{-3} \leqslant \varepsilon_{k} \leqslant 10^{6},-150 \leqslant x_{k} \leqslant 150$, $-100 \leqslant y_{k} \leqslant 100(k=1, \ldots, s)$. The values of the parameters $Q^{(p)}, \psi_{* j}^{(p)}, \psi_{j}^{*(p)} \bar{\varphi}_{i}^{(p)}, \underline{\varphi}_{i}^{(p)}, s, \chi, \alpha_{k}, \varepsilon_{k}$, $x_{k}, y_{k}(k=1, \ldots, s)$ are given if there is a possibility of estimating the accuracy of the algorithm [21] in the way of the corresponding comparisons (numerical calculations of characteristic parameters and given reference standards values).
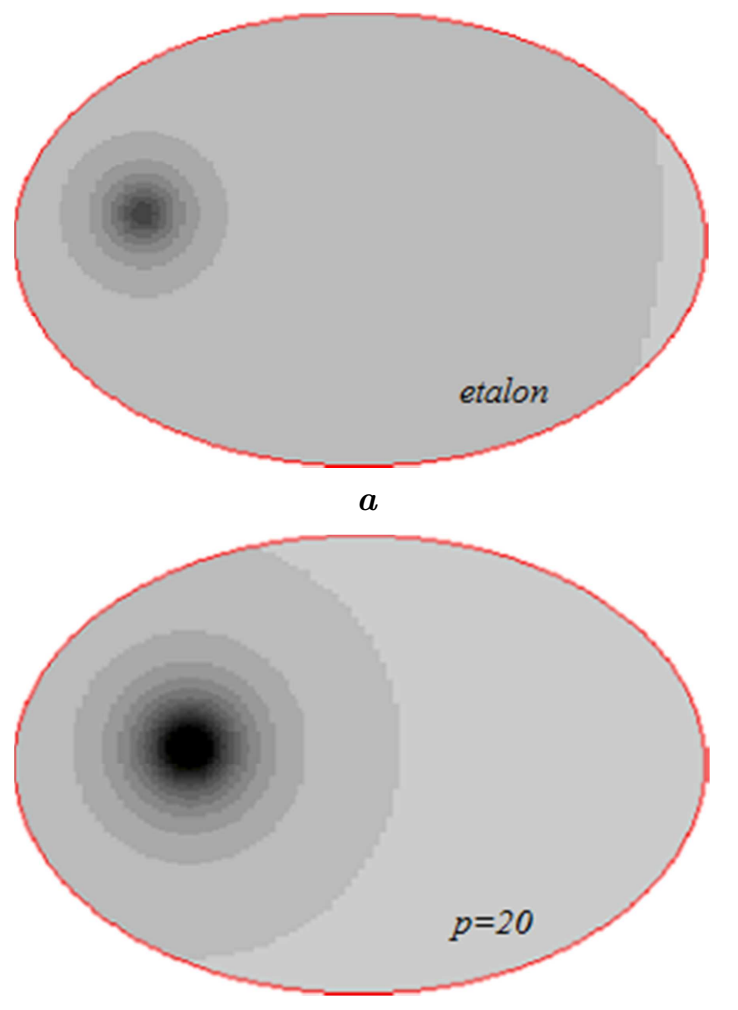

c
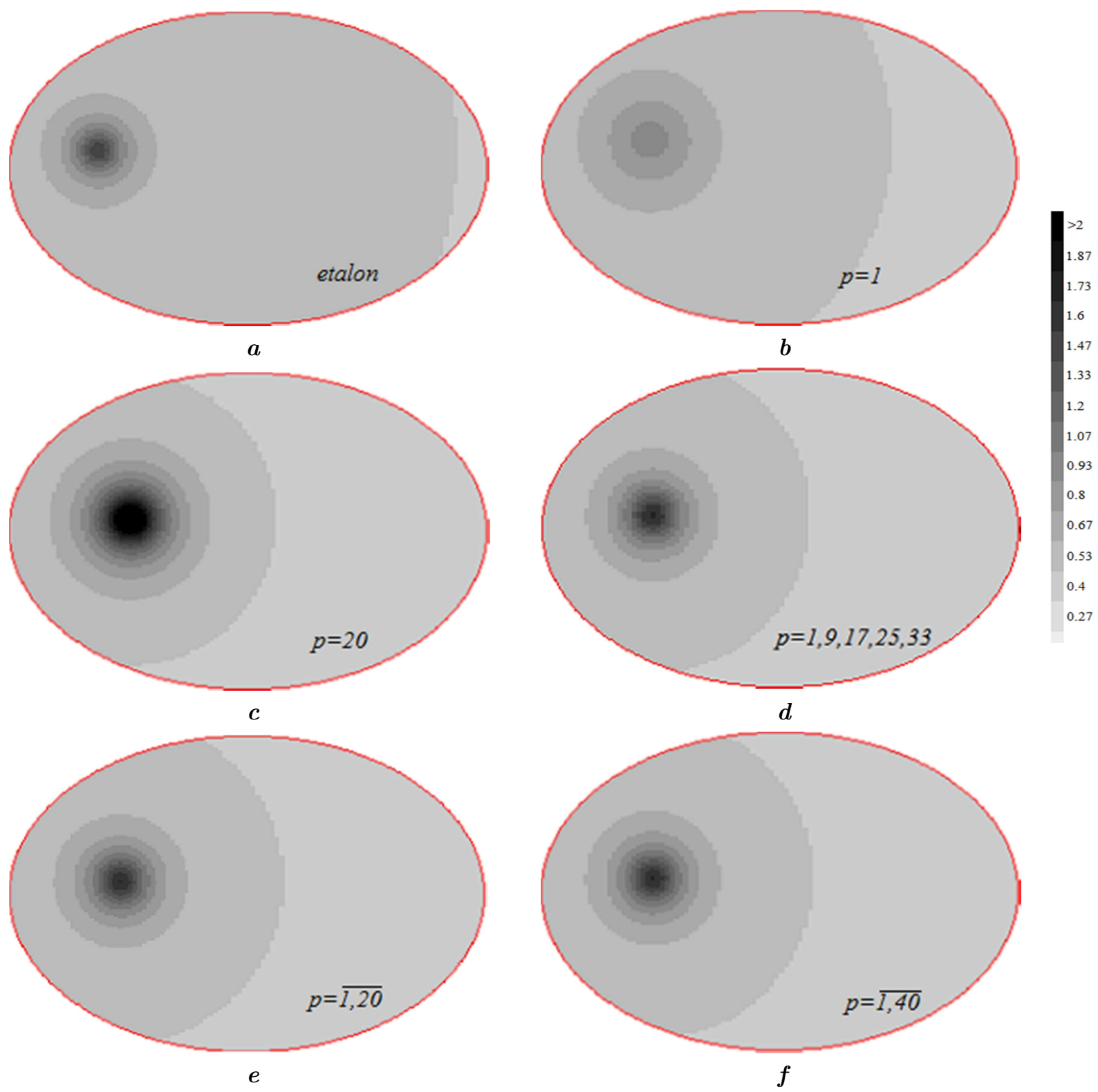

Fig. 2. Distributions of the CC for the example with one local burst.

In Fig. $2 a$, an etalon is shown for $s=1, \chi=0.5, \alpha_{1}=1, \varepsilon_{1}=200, x_{1}=-95, y_{1}=-12$. And in Table 1 and Fig. $2 b-2 e$, the results of calculations are shown for the characteristic parameters $\Phi, \chi$, $\alpha_{1}, \varepsilon_{1}, x_{1}, y_{1}$ and under the condition that only the first of the forty injections $(\tilde{p}=40)$ is performed, for the twentieth injection, for first twenty injections, and for all forty injections. We can note that 
a certain increase in the total number of injections significantly improves the reconstruction of the image in comparison with the results of the individual injections, but performing too much of them, as expected, has no meaning (the results of the reconstruction of five, twenty, and forty injections are almost coincident).

Table 1. Distributions of the CC for the example with one local burst.

\begin{tabular}{|c|c|c|c|c|c|c|c|}
\hline & $p$ & $\Phi$ & $\chi$ & $\alpha_{1}$ & $\varepsilon_{1}$ & $x_{1}$ & $y_{1}$ \\
\hline$a$ & - & - & 0.5 & 1 & 200 & -95 & -12 \\
\hline$b$ & 1 & 2.4966 & 0.4857 & 0.4495 & 993.0104 & -82.576 & -17.6735 \\
\hline$c$ & 20 & 6.6754 & 0.4421 & 1.9348 & 278.815 & -74.9674 & -7.5969 \\
\hline$d$ & $1,9,17,25,33$ & 28.498 & 0.4722 & 1.198 & 267.8533 & -81.2635 & -8.7357 \\
\hline$e$ & $1,2,3, \ldots, 20$ & 98.7319 & 0.4744 & 1.1712 & 274.7286 & -80.7618 & -8.4726 \\
\hline$f$ & $1,2,3, \ldots, 40$ & 220.3432 & 0.4727 & 1.1719 & 278.497 & -80.7184 & -8.7694 \\
\hline
\end{tabular}

Table 2 and Fig. 3 demonstrate the results of calculations and the reconstruction of images in the presence of two and three bursts of the CC for the corresponding reference standards values $s=2$, $\chi=0.5, \alpha_{1}=1, \varepsilon_{1}=200, x_{1}=-95, y_{1}=-12, \alpha_{2}=0.5, \varepsilon_{2}=800, x_{2}=0, y_{2}=50 ; s=3, \chi=0.5$, $\alpha_{1}=1, \varepsilon_{1}=200, x_{1}=-80, y_{1}=30, \alpha_{2}=0.5, \varepsilon_{2}=1000, x_{2}=50, y_{2}=50, \alpha_{3}=0.3, \varepsilon_{3}=2000$, $x_{3}=0, y_{3}=-80$.

Table 2. Distributions of the CC for the examples of two (a, b) and three (c, d) local bursts.

\begin{tabular}{|c|c|c|c|c|c|c|c|c|c|c|c|c|c|c|c|}
\hline & $s$ & $\Phi$ & $\chi$ & $\alpha_{1}$ & $\varepsilon_{1}$ & $x_{1}$ & $y_{1}$ & $\alpha_{2}$ & $\varepsilon_{2}$ & $x_{2}$ & $y_{2}$ & $\alpha_{3}$ & $\varepsilon_{3}$ & $x_{3}$ & $y_{3}$ \\
\hline$a$ & 2 & - & 0.5 & 1 & 200 & -95 & -12 & 0.5 & 800 & 0 & 50 & - & - & - & - \\
\hline$b$ & 2 & 132 & 0.22 & 0.56 & 10505 & 11 & 24 & 0.84 & 509 & -96 & -10 & - & - & - & - \\
\hline$c$ & 3 & - & 0.5 & 1 & 200 & -80 & 30 & 0.5 & 1000 & 50 & 50 & 0.3 & 2000 & 0 & -80 \\
\hline$d$ & 3 & 157 & 0.21 & 0.5 & 1852 & -88 & 17 & 0.57 & 8944 & 47 & 31 & 0.3 & 2487 & -6 & -84 \\
\hline
\end{tabular}
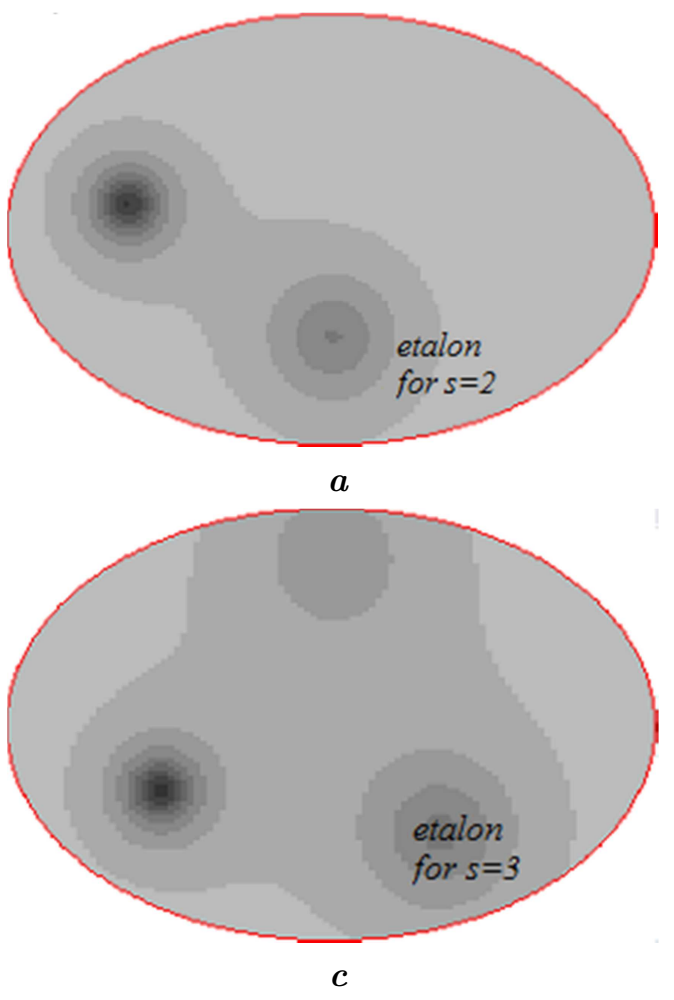

Fig. 3. Distributions of the $\mathrm{CC}$ for the examples with two $(a, b)$ and three $(c, d)$ local bursts. 
Note that here (for the simplification of the statements) were considered the bursts of homogeneities (the procedure for the reconstruction of bursts of well-studied heterogeneous media can be similarly described). We also note that the developed algorithm is sufficiently open for various additions, generalizations, etc., in particular, if the process is unstable, there is a possibility, as in [1,7], to "introduce" the regularization procedure of the Tikhonov type, when instead of (8), the functional is considered

$$
\begin{aligned}
& \Phi\left(x^{(1)}, \ldots, x^{(\tilde{p})}, y^{(1)}, \ldots, y^{(\tilde{p})}, \chi, \alpha_{1}, \ldots, \alpha_{s}, \varepsilon_{1}, \ldots, \varepsilon_{s}, x_{1}, \ldots, x_{s}, y_{1}, \ldots, y_{s}\right) \\
& \quad=\sum_{p=1}^{\tilde{p}}\left(\sigma\left(x^{(p)}, y^{(p)}, \chi, \alpha_{1}, \ldots, \alpha_{s}, \varepsilon_{1}, \ldots, \varepsilon_{s}, x_{1}, \ldots, x_{s}, y_{1}, \ldots, y_{s}\right)-f\left(x^{(p)}, y^{(p)}\right)\right)^{2} \\
& +\eta \cdot \Omega\left[x^{(1)}, \ldots, x^{(\tilde{p})}, y^{(1)}, \ldots, y^{(\tilde{p})}, \chi, \alpha_{1}, \ldots, \alpha_{s}, \varepsilon_{1}, \ldots, \varepsilon_{s}, x_{1}, \ldots, x_{s}, y_{1}, \ldots, y_{s}\right] \rightarrow \min ,
\end{aligned}
$$

where $\Omega\left[x^{(1)}, \ldots, x^{(\tilde{p})}, y^{(1)}, \ldots, y^{(\tilde{p})}, \chi, \alpha_{1}, \ldots, \alpha_{s}, \varepsilon_{1}, \ldots, \varepsilon_{s}, x_{1}, \ldots, x_{s}, y_{1}, \ldots, y_{s}\right]$ is the stabilizing functional, $\eta$ is the parameter of regularization.

\section{Conclusions}

In the article a numerical method of quasiconformal mappings is developed for the identification of parameters of local bursts of the coefficient of conductivity of the medium according to the data of the tomography of the applied quasipotentials, based on which the problem of analysis in the iterative process is reduced to the application of numerical methods of quasiconformal mappings, and the problem of synthesis is reduced to the solving the parametric identification problem. The use of a specially developed procedure for "averaging" intermediate iterative approximations of the piecewise homogeneous CC according to the data on all injections is extended over the case of the inhomogeneous medium.

We see that, as it should be expected, for a qualitative reconstruction of an image (identification of five parameters of the burst), it is necessary, generally speaking, to carry out five injections, although previous (rough reconstruction) can be obtained even with one injection (under condition of successful application of potentials).

An essential feature of the developed algorithm is the possibility of its comparatively easy parallelization and stopping of the calculation procedure if only some of the conditions for the completion of the process hold with the automatic determination of those parts of the physical area where large errors of computations occur, what makes it possible to use the computing time more economically. The latter is relevant, in particular, in the case of the so-called "dead zones" and "zones of large gradients" that arise near the special angular points of smooth boundary lines of the corresponding domains.

For the future, we plan to extend the algorithm over the spatial case and the cases of the presence of several areas of application of potentials.

[1] Bomba A. Ya., Kroka L. L. Tchysloviy metod kvazikonformnogo vidobrazhennya rozv'yazannya zadach identifikatsii koyefitsienta elektrichnoi providnosti za danymy tomografii prykladenikh potentsialiv. Volyns'kiy matematychniy visnyk. Seriya prykladna matematika. 11(20), 24-33 (2014), (in Ukrainian).

[2] Bomba A. Ya., KrokaL.L. Chislovi metody kompleksnogo analizu pry rozv'yazanni odnogo klasu neliniynikh eliptichnikh zadach za umov identifikatsii parametriv. Matematychne ta komp'yuterne modelyuvannya. Seriya: Fizyko-matematychni nauki: zb. nauk. prats'. 10, 24-33 (2014), (in Ukrainian).

[3] Bomba A. Ya., Boichura M. V. One numerical complex analysis method for parameters identification of piecewise homogeneous conductivity media with using applied quasipotential tomographic data. Matematychne ta komp'yuterne modelyuvannya. Seriya: Tekhnichni nauki: zb. nauk. prats'. 14, 5-17 (2016). 
[4] Gorb M. S., Gusieva O. V. Vybir matematychnoi modeli ob'yektu doslidzhennya v elektroimpedansniy tomografi. Visnyk NTUU "KPI". Seriya Radiotekhnika. Radioaparatobuduvannya. 52, 120-128 (2013), (in Ukrainian).

[5] Sushko H. O., Rybin O. H. Vizualizatsiya rozpodilu poverkhnevykh providnostey metodom zon providnosti. Visnyk NTUU "KPI". Seriya Radiotekhnika. Radioaparatobuduvannya. 5, 7-17 (2014), (in Ukrainian).

[6] Gavrilov S.V. Iteratsionnyy metod resheniya trekhmernoy zadachi elektroimpedansnoy tomografii v sluchaye kusochno-postoyannoy provodimosti i neskol'kikh izmereniy na granitse. Vychislitel'nyye metody i programmirovaniye: novyye vychislitel'nyye tekhnologii. 14(1), 26-30 (2013), (in Russian).

[7] Sherina Ye. S., Starchenko A. V. Chislennoye modelirovaniye zadachi elektroimpedansnoy tomografii i issledovaniye podkhoda na osnove metoda konechnykh obyemov. Byulleten' sibirskoy meditsiny. 4, 156-164 (2014), (in Russian).

[8] Denyer C. W. L. Electronics for Real-Time and Three-Dimensional Electrical Impedance Tomographs: PhD Thesis. Oxford (1996).

[9] Holder D. Electrical Impedance Tomography. Methods, History and Applications. London, Institute of Physics (2005).

[10] Hou T. C., Lynch J. P. Electrical Impedance Tomographic Methods for Sensing Strain Fields and Crack Damage in Cementitious Structures. Journal of Intelligent Material Systems and Structures. 20, 13631379 (2009).

[11] Haddar H., Kress R. Conformal Mapping and an Inverse Impedance Boundary Value Problem. Journal of Inverse and Ill-posed Problems. 14(8), 785-804 (2006).

[12] Liu D. Kolehmainen V., Siltanen S. and others. Estimation of Conductivity Changes in a Region of Interest with Electrical Impedance. Inverse Problems and Imaging. 9(1), 211-229 (2014).

[13] Sun T. Tsuda S., Zauner K. and others. On-chip electrical impedance tomography for imaging biological cells. Biosensors and Bioelectronics. 25(5), 1109-1115 (2010).

[14] Bomba A. Ya., Kashtan S. S., Prigornits'kiy D. O., Yaroshchak S. V. Metody kompleksnogo analizu: monografiya. Rivne, NUVGP (2013), (in Ukrainian).

[15] Bomba A. Ya., Bulavats'kiy V. M., Skopets'kiy V. V. Neliniyni matematichni modeli protsesiv geogidrodinamiki. Kyiv, Naukova dumka (2007), (in Ukrainian).

[16] Lavrent'yev M. A., ShabatB.V. Metody teorii funktsii kompleksnogo peremennogo. Moscow, Nauka (1973), (in Russian).

[17] Somersalo E., Cheney M., Isaacson D. Existence and uniqueness for electrode models for electric current computed tomography. SIAM J. Appl. Math. 52(4), 1023-1040 (1992).

[18] Ortega J. M., Rheinboldt W. C. Iterative Solution of Nonlinear Equations in Several Variables. Classics in Appl. Math. SIAM Publications, Philadelphia, 2nd edition (2000).

[19] e Oliveira H. A. Jr., Ingber L., Petraglia A. and others. Stochastic Global Optimization and Its Applications with Fuzzy Adaptive Simulated Annealing. Heidelberg, Springer-Verlag (2012).

[20] Samarskiy A. A. Teoriya raznostnykh skhem. Moscow, Nauka (1977), (in Russian).

[21] Sherina Ye.S., Starchenko A.V. Raznostnyye skhemy na osnove metoda konechnykh ob'yomov dlya zadachi elektroimpedansnoy tomografii. Vestnik tomskogo gosudarstvennogo universiteta. Matematika i mekhanika. 3(29), 25-38 (2014), (in Russian). 


\title{
Числовий метод квазіконформних відображень ідентифікації параметрів середовищ за даними томографії прикладених квазіпотенціалів
}

\author{
Бомба А., Бойчура М. \\ Рівненсъкий державний гуманітарний університет \\ вул. С. Бандери, 12, 33000, Рівне, Україна
}

Розглянуто задачу ідентифікації параметрів сплесків коефіцієнта провідності середовища за даними томографії прикладених квазіпотенціалів. Запропоновано метод реконструкції зображення, згідно з яким задача аналізу зводиться до застосування числових методів квазіконформних відображень, а задача синтезу - до розв'язання задачі параметричної ідентифікації. Наведено результати числових експериментів та проведено їх аналіз.

Ключові слова: томографія прикладених квазіпотенціалів, квазіконформні відображення, ідентифікація, нелінійні задачі.

2000 MSC: 630C30; 30C62

УдК: 519.6 